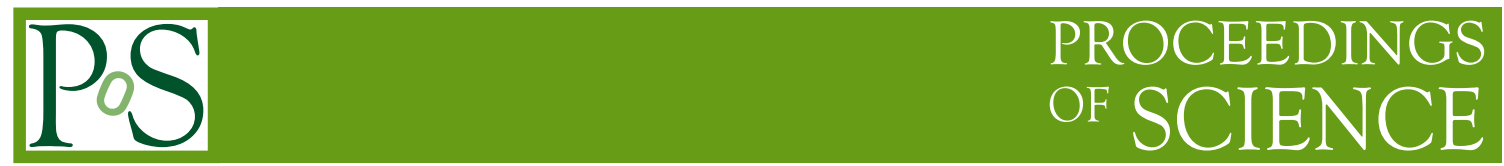

\title{
LHCb Run 2 trigger and upgrade reconstruction
}

\section{Mark Whitehead*广}

RWTH Aachen University

E-mail: mwhitehe@cern.ch

The LHCb trigger strategy for Run II (2015-2018) and the upgrade era from Run III (2021) onwards are discussed. A crucial aspect of the LHCb upgrade is the removal of the hardware trigger, so LHCb will move to a fully software based trigger. The latest studies and progress from the upgrade tracking and reconstruction sequence, that will form the basis of the upgrade trigger, are presented.

The 39th International Conference on High Energy Physics (ICHEP2018)

4-11 July, 2018

Seoul, Korea

* Speaker.

${ }^{\dagger}$ On behalf of the LHCb collaboration. 


\section{Introduction}

The LHCb detector will be upgraded during long shutdown two of the LHC during 2019 and 2020. A major part of the upgrade is the removal of the level 0 hardware trigger, so LHCb will move to a fully software based trigger. LHCb currently runs at a luminosity of $\mathscr{L} \approx 4 \times 10^{32} \mathrm{~cm}^{-2} \mathrm{~s}^{-1}$, around a factor of 10 below the nominal instantaneous luminosity of the LHC, due to design choices based on the physics programme and detector and trigger limitations. From Run III, LHCb will run at a luminosity of $\mathscr{L} \approx 2 \times 10^{33} \mathrm{~cm}^{-2} \mathrm{~s}^{-1}$, increasing the size of the collected data samples by a factor of five from the luminosity and up to an additional factor of two from the increased efficiency after removing the hardware trigger. Note that this update takes place before the high luminosity LHC project. These proceedings describe the trigger strategy for Run II (2015-2018) and the progress towards Run III (2021-2023).

\section{Run II strategy}

The LHCb trigger strategy during Run II is shown in Fig. 1 (left). It features a hardware trigger that reduces the rate from $40 \mathrm{MHz}$ to $1 \mathrm{MHz}$ using information from the calorimeters and muon stations. The software trigger is split into two stages, high level trigger (HLT) 1 and 2, with HLT1 reducing the rate to around $110 \mathrm{kHz}$ before HLT2 reduces the output rate to about $12.5 \mathrm{kHz}$ before sending data to storage. The disk buffer between the HLT1 and HLT2 stages enables online detector calibration and alignment, which in turn facilitates performing physics analyses directly from the trigger [1] output. An example of the real-time alignment of the VELO is shown in Fig. 2 for a period in April and May 2018, showing the updates that were made on a fill-by-fill basis.

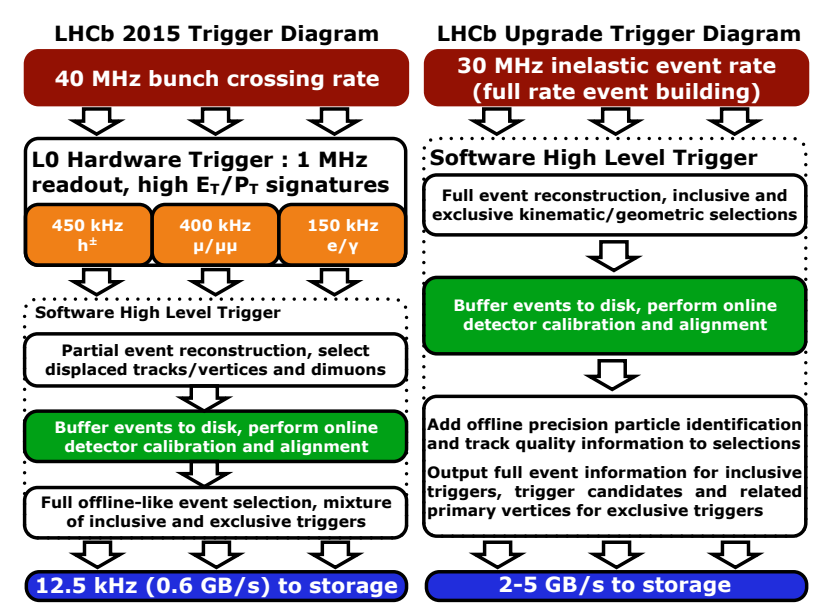

Figure 1: The LHCb trigger strategy for (left) Run II and (right) the upgrade era.

The 10 PB disk buffer provides a two week contingency when filled at $110 \mathrm{kHz}$ by HLT1 with an average event size of $55 \mathrm{kB}$, effectively doubling the available CPU resources. During a fill HLT1 runs at $110 \mathrm{kHz}$ and the remaining CPU resources are taken by HLT2, outside of fills HLT2 is given $100 \%$ of the CPU resources. 


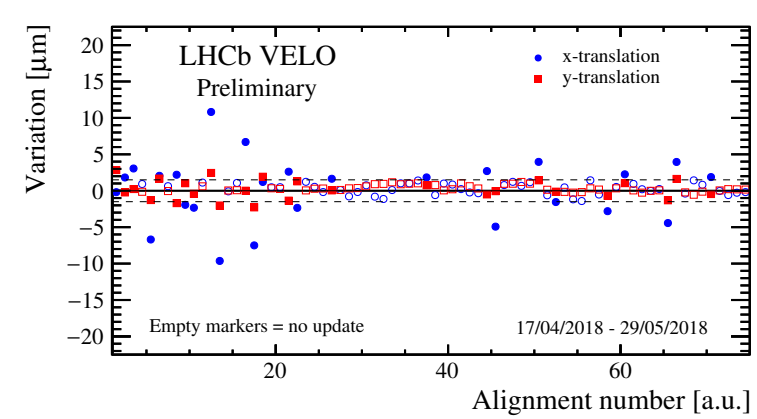

Figure 2: Real-time alignment of the LHCb vertex locator (VELO) subdetector performed each fill for a period during spring 2018. The points are as described by the legend.

\section{LHCb upgrade trigger}

The LHCb upgrade era will see a change in the trigger paradigm, 24\% (2\%) of bunch crossings will contain a charm (beauty) hadron. Therefore, the trigger must separate signal from backgrounds, but also signal decays from other signal decays. Additionally, it must be flexible to cater for high rate charm decays and rare beauty decays together. The Turbo stream paradigm will be the way forward, focussing on a reduction of the size on disk for each event to allow more to be saved for the same output bandwidth. Increased pile-up of events poses an additional challenge, moving from around 1.2 to 6 interactions per bunch crossing. As seen in Fig. 1, the hardware trigger will be removed while the strategy for the software trigger remains similar. The upgrade HLT1 stage includes data preparation, track reconstruction and efficient selections to reduce the rate to between 0.5-1.0 MHz. The real-time and alignment strategy from Run II will be followed. Finally, HLT2 will perform the full event reconstruction, including the best tracking performance with the addition of PID information and offline quality selections.

\subsection{Upgrade tracking and reconstruction}

The upgrade tracking and reconstruction includes a fast stage in HLT1 for preliminary selections and online alignment and calibration. The second, best, stage performs the rest of the reconstruction in HLT2. Some recent studies are summarised in the following.

Data preparation currently uses around half of the timing budget in HLT1. This includes decoding raw data and performing clustering for the VELO. This forms a global optimisation, fast algorithms are crucial but the data must also be prepared as efficiently as possible. Several improvements have been made and profiling is underway to highlight further areas to study.

The throughput (the number of events processed per second, per node) of the HLT1 tracking sequence is being consistently improved, including a gain of more than a factor of two over the last year. Moving new multi-threading framework shows a $20 \%$ improvement, as shown by Fig. 3.

The Kalman filter used in the track fit also uses a significant part of the HLT1 timing budget. In Run I a material look-up and $B$ field propagation were performed, in Run II the full material map was replaced by a simplified model to speed up the execution time. In Run III a parameterised Kalman filter is under study, replacing the material and $B$ field maps with analytic functions. This is much faster than a full Kalman filter and already shows excellent performance, as shown by Fig. 4. 


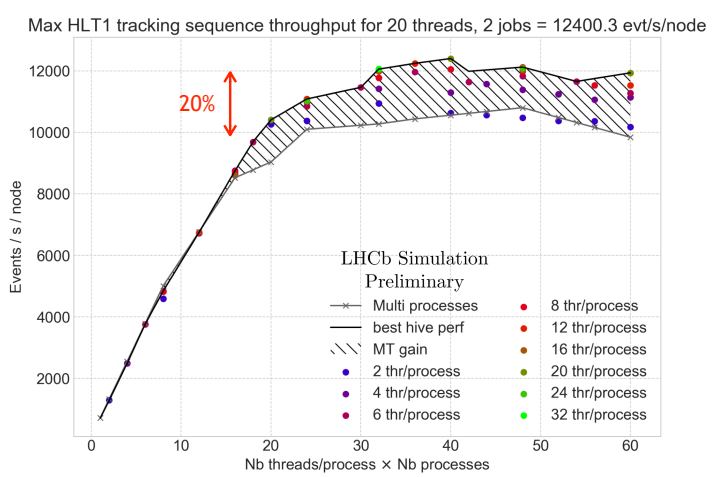

Figure 3: Throughput of the HLT1 tracking sequence comparing between multi-threaded and singlethreaded frameworks. The shaded band shows the $\approx 20 \%$ gained by moving to the new multi-threading framework. Figure reproduced from Ref. [2].

Here the parameterised Kalman (red) shows very similar performance in terms of momentum resolution with respect to the full Kalman filter shown in black.

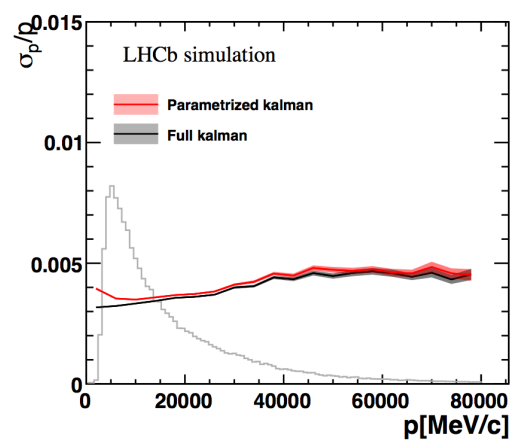

Figure 4: Comparison of the momentum resolution of the parameterised (red) and full (black) Kalman filter as a function of track momentum. The grey histogram shows the distribution of track momenta.

\section{Summary}

The strategy of the LHCb Run II trigger is shown as a pathway to the upgrade trigger. The latest developments in the upgrade tracking and reconstruction are shown. The upgrade trigger is a real challenge and great progress is being made. The HLT1 fast tracking sequence is evolving rapidly, with an equal focus on speeding up algorithms and data preparation. Detailed profiling is underway to highlight areas for further improvement. The efficiency for beauty and charm decays is already better than seen in Run II, and this will be pushed and developed further over the next two years.

\section{References}

[1] R. Aaij et al., Tesla : an application for real-time data analysis in High Energy Physics, Comput. Phys. Commun. 208 (2016) 35-42.

[2] LHCb Collaboration, Upgrade Software and Computing, CERN-LHCC-2018-007, 2018. 